\title{
A Sustentabilidade em Cadeias de Suprimento a partir da visão de recursos e capacidades
}

\section{Sustainable Supply Chain Management from resources and capabilities}

\author{
Daiane Mulling Neutzling ${ }^{1}$ \\ Minelle Enéas da Silva ${ }^{2}$
}

\section{Resumo}

Diante das diversas demandas ligadas à inserção de sustentabilidade em estratégias organizacionais, aquelas relacionadas com cadeias de suprimento têm emergido como centrais. Nesse contexto, as organizações têm possibilidades de desenvolver estratégias socioambientais a partir de seus recursos e das iniciativas focadas em suas fronteiras organizacionais. Contudo, quando essas estratégias são extrapoladas para as cadeias de suprimento, resultados mais satisfatórios podem ser alcançados no que se refere à utilização de recursos e capacidades utilizados para o desenvolvimento de relacionamentos interorganizacionais. Com essa perspectiva, o presente estudo tem como objetivo compreender como recursos e capacidades podem facilitar a efetivação de Sustentabilidade em Cadeias de Suprimento (SCS), a partir da Visão Baseada em Recursos (VBR) e da Visão Relacional (VR). Por meio de um ensaio teórico, buscou-se debater como tais visões podem servir como base para alguns direcionamentos práticos em relação a como empresas podem se utilizar de recursos e capacidades para desenvolver estratégias sustentáveis que podem ser introduzidos pelas empresas e ao longo das cadeias de suprimentos. As discussões demonstram que, ao relacionar a VBR e VR à SCS, pode-se ter uma oportunidade de explicar melhor como elementos organizacionais internos à empresa, mediante mecanismos de governança apropriados, podem ser integrados em relacionamentos colaborativos nas cadeias de suprimento. Além disso, apresentam potenciais ações organizacionais que podem levar à criação de

Doutora em Administração pela UFRGS. Professora no PPGA (UNIFOR) - Brasil - E-mail: daineutzling@gmail.com

2 Doutor em Administração pela UFRGS. Professor no PPGA (UNIFOR) - Brasil - E-mail: minele. adm@gmail.com 
vantagens competitivas e/ou colaborativas. Portanto, este artigo contribui para a construção de literatura, abordando o desenvolvimento de recursos e capacidades quanto à Sustentabilidade em Cadeias de Suprimento.

Palavras-chave: Sustentabilidade. Cadeias de Suprimento. Estratégia. Recursos. Capacidades.

\section{Abstract}

Due to asetofdemands related to the sustainability inclusion in organizational strategies those related to supply chains have emerged as central. In this context, organizations have possibilities to develop sustainability strategies from their resources and initiatives focused on their organizational boundaries. However, when these strategies are extended to supply chains more effective results can be achieved by using internal resources and capabilities for developing to interorganizational relationships. Therefore, this study aims to understand how resources and capabilities can facilitate the application of sustainable supply chain management from the perspective of the Resource Based View (RBV) and Relational View (RV). Thus, a theoretical essay was developed in order to present how those perspectives can be used as a base to propose some practical directions for companies to use resources and capabilities to develop sustainable strategies along the supply chain. Therefore, we present that the perspectives of RBV and RV integrated to the context of sustainable supply chain management can bean opportunity to explore howto integrate internal organizational elements into collaborative relationships in supply chains through appropriate governance mechanisms. Also, presents potential organizational actions that can lead to the creation of competitive and / or collaborative advantages. In this context, this paper contributes to the literature improvement to address the development of resources and capabilities in relation to sustainable supply chain management.

Keywords: Sustainability. Supply Chains. Strategy.Resources. Capabilities.

\section{Introdução}

Ao longo do tempo, continuamente, o ambiente organizacional tem vivenciado um conjunto de transformações advindas de políticas governamentais, legislações, pressões da sociedade e de instituições do terceiro setor. Tais mudanças têm suas origens na intensificação das discussões em torno da sustentabilidade e dos novos comportamentos e práticas que são exigidos na sociedade. Uma dessas transformações está associada à relevância de desenvolver estratégias que considerem 
as dimensões do Triple Bottom Line (TBL) (ambiental, econômica e social) nos negócios corporativos (Elkington, 1999).

A criação de novas estratégias, a partir de uma discussão socioambiental, tem em uma de suas bases a responsabilidade social (e ambiental) em relação ao contexto da empresa (Sousa Filho\& Barbieri, 2015). Nesse sentido, ressalta-se a capacidade que as empresas possuem em transformar seus recursos financeiros, conhecimento tecnológico e a atuação institucional em desenvolvimento de processos produtivos que contribuam para a equidade social e a proteção ambiental (Pagell \& Wu, 2009; Shrivastava, 1995). Ainda, as preocupações relacionadas à redução dos impactos socioambientais passaram a ser tidas como determinantes de ganhos competitivos (Carter \& Rogers, 2008; Porter \& Kramer, 2006).

Levar em consideração as questões ambientais e sociais pode facilitar a otimização de recursos e ao aumento das capacidades organizacionais (Silva \& Balbino, 2013), fato que deve considerar tanto as potencialidades internas como aquelas aprendidas em meio a relações de mercado. Surgem, assim, oportunidades para diferenciação de produtos e serviços baseados nas dimensões avaliadas e também na construção de uma reputação corporativa frente à mídia e aos órgãos governamentais (Bansal, 2005; Porter \& Kramer, 2006; Rao \& Holt, 2005). Isso ocorre no sentido de melhor posicionar as empresas à sustentabilidade.

Segundo Paulraj (2011), ao desenvolver estratégias que integram os valores da sustentabilidade aplicados aos modelos de negócios, as organizações criam uma situação de valor compartilhado com a sociedade que pode lhes proporcionar uma condição de se capitalizar nos mercados e serem detentoras de vantagens iniciais (first movers). Assim, ao se integrar as dimensões do TBL, pode-se influenciar na construção de uma base sólida de rentabilidade e crescimento, determinantes de competitividade (Carter \& Rogers, 2008; Pagell \& Wu, 2009; Paulraj, 2011; Seuring \& Müller, 2008). Além disso, essa nova posição estratégica facilita o surgimento de vantagens competitivas sustentáveis. 
Nesse contexto, outra questão relevante a ser destacada, refere-se aos limites de atuação das empresas e à aplicação de suas estratégias socioambientais. As organizações têm possibilidades de desenvolver estratégias de sustentabilidade por meio de seus recursos internos e das iniciativas focadas em suas fronteiras organizacionais, contudo, quando estas estratégias são extrapoladas para as suas cadeias de suprimento, resultados mais satisfatórios podem ser alcançados (Carter \& Rogers, 2008; Srivastava, 2007). Portanto, ao se buscar a Sustentabilidade em Cadeias de Suprimento (SCS), considera-se que o elo ou agente que gerencia o relacionamento pode gerar uma nova interação entre os membros e desenvolver comportamentos colaborativos a fim de promover a sustentabilidade ao longo de sua cadeia.

A proposta de Sustentabilidade em Cadeias de Suprimento está relacionada a uma orientação das organizações em busca de novos comportamentos pela integração de estratégias de suprimento e de sustentabilidade nas estratégias corporativas (Beske, 2012; Seuring \& Müller, 2008). O foco seria a identificação de ameaças e oportunidades, considerando que a gestão estratégica da cadeia de suprimento voltada à sustentabilidade estaria ligada a uma perspectiva de mudanças de processos, adequações legais e de fortalecimento da relação com clientes e fornecedores em longo prazo, visando compartilhar recompensas e riscos (Bowen et al., 2002; Preuss, 2006; Srivastava, 2007).

Dessa forma, defende-se que, ao estender a orientação de sustentabilidade à cadeia de suprimento, pode-se alcançar não somente a minimização dos impactos ambientais e sociais, como desenvolver recursos e capacidades diferenciados a partir de relacionamentos colaborativos entre os parceiros dessa cadeia. A trajetória das empresas e seu conhecimento prévio, quando incorporados ao conhecimento e recursos dos parceiros, podem gerar recursos e competências idiossincráticos, além do gerenciamento dos riscos e incertezas, gerando vantagens competitivas e/ou colaborativas para a empresa focal e também de seus fornecedores, através das rendas relacionais obtidas (Dyer \& Singh, 1998; Gold et al., 2010; Markley \& Davis, 2007; Paulraj, 2011; Rao \& Holt, 2005; Zacharia et al., 2011). 
Como explicitado pela Visão Baseada em Recursos (VBR) (Amit \& Schoemaker, 1993; Barney, 1991; Peteraf, 1993; Wernerfelt, 1984) e pela Visão Relacional (VR) (Duschek, 2004; Dyer \& Singh, 1998), os recursos necessitam ser configurados como socialmente complexos, ambiguamente causais e particularmente difíceis de serem imitados pelos concorrentes. Assim, entender essas abordagens e seus reflexos nas estratégias das empresas pode facilitar o entendimento da importância de estratégias envolvendo a sustentabilidade.

Alguns trabalhos científicos que abordam a sustentabilidade no contexto da gestão de cadeias de suprimento (GCS) já têm apontado de forma exploratória, para a importância destes recursos e capacidades nesse processo (Chen \& Paulraj, 2004; Gold et al., 2010; Vachon \& Klassen, 2008). Partindo dessa contextualização inicial, a problemática discutida neste artigo se refere a: de que forma recursos e capacidades internos das empresas, alinhados aos recursos interorganizacionais, podem proporcionar meios para que a sustentabilidade seja integrada à gestão da cadeia de suprimento?

Desse modo, o objetivo é compreender como recursos e capacidades podem facilitar a efetivação da Sustentabilidade em Cadeias de Suprimento, a partir da Visão Baseada em Recursos e da Visão Relacional. Justifica-se a presente pesquisa a partir de dois aspectos centrais: (1) poucas pesquisas no Brasil estão aproximando as linhas de estudo aqui debatidas; e (2) a presente discussão pode ser suporte para um maior aprofundamento teórico em SCS, no sentido de propor uma nova forma de pesquisa ao tema.

O presente estudo caracteriza-se como um ensaio teórico à medida que não se restringe a analisar o que a literatura versa sobre o assunto, mas realizar uma escrita reflexiva para estabelecer relações, convergir pensamentos e propor questionamentos que enriquecem o debate do assunto (Meneghetti, 2011). Para uma compreensão mais clara da proposta, a seção seguinte apresenta as discussões teóricas sobre o tema, com foco na Sustentabilidade em Cadeias de Suprimento, e os principais elementos para o desenvolvimento de recursos e 
capacidades, segundo a VBR e a VR. A terceira seção traz a proposta de conversão entre os temas, com discussões sobre o que contribui para a sustentabilidade em cadeias de suprimento (SCS). E, por fim, as considerações finais, com as contribuições do estudo.

\section{Discussão teórica}

\subsection{Sustentabilidade em Cadeias de Suprimento: uma macro visão}

O desenvolvimento da sustentabilidade por uma vertente empresarial se dá principalmente pelo trabalho de John Elkington, ao propor que os negócios estabelecidos de forma responsável deveriam se basear em um tripé, considerando, além dos lucros, a responsabilidade com as pessoas e com o meio ambiente (ELKINGTON, 1999). Esse conceito se tornou conhecido como Triple Bottom Line (TBL) e tem sido amplamente disseminado.

O TBL também foi inserido na literatura da gestão de cadeias de suprimento, dando origem ao Sustainable Supply Chain Management (SSCM). Este conceito se torna de fato conhecido e explorado a partir de 2008, com os estudos de Seuring e Müller (2008) e Carter e Rogers (2008) (Ahi \& Searcy, 2013; Ashby et al., 2012). A tradução para o português ainda possui alguns conflitos e incertezas (Silva et al., 2015), mas a discussão aqui realizada assume a expressão "Sustentabilidade em Cadeias de Suprimento".

Tal conceito visa discutir a gestão eficiente e transparente de processos ao longo das cadeias, considerando tanto aspectos econômicos e de competição quanto as questões socioambientais envolvidas neste contexto. Assim, a SCS deve ser analisada não somente pelo seu desempenho econômico, mas também por seu desempenho socioambiental (Beske, 2012). Corroborando com essa perspectiva, Pagell e Schevchenko (2014) indicam que a gestão da cadeia de suprimento deve considerar de forma equilibrada tanto o desempenho 
econômico quanto o social e o ambiental. O conceito mais disseminado, até então, sobre o tema indica que este se refere à:

Gestão de fluxos de capital, materiais e informações, bem como, a cooperação entre as empresas ao longo da cadeia de suprimentos, visando metas para as três dimensões: econômica, ambiental e social, que são requisitos dos clientes e stakeholders. (Seuring\& Müller, 2008, p. 1700).

A evolução do conceito possui algumas linhas de pensamento que, de forma ainda não convergente em sua totalidade, tanto aproxima a visão tradicional da cadeia de suprimento com a sustentabilidade como busca estimular o desenvolvimento de uma perspectiva mais flexível para entender o relacionamento entre cadeias de suprimento e sustentabilidade por meio de um alinhamento de estratégias, ao invés de uma sobreposição ou uma inserção por si (Beske \& Seuring, 2014; Halldórsson et al., 2009; Pagell \& Wu, 2009). Apesar disso, Golicic e Smith (2013) afirmam que o estudo do tema é oportuno e significativo de ser realizado, uma vez que traz à tona uma discussão que transborda as fronteiras de uma empresa e considera os atores que formam sua cadeia de suprimento.

Vale salientar que o conceito de cadeia de suprimento aqui utilizado - e o disseminado com mais frequência em estudos sobre o tema - é aquele apresentado por Mentzer et al. (2001), no qual os autores trazem a perspectiva de posicionamento estratégico e gestão que essa forma de relacionamento interorganizacional traz entre a empresa focal e os demais membros da cadeia de suprimento. Além disso, entendese que não existe a exigência de uma lógica linear e sequencial entre os participantes, mas que a formação desta é dimensionada a partir daqueles que a compõe e da maneira como estes interagem. Para os autores, a gestão da cadeia de suprimento é:

A coordenação sistemática e estratégica das funções empresariais tradicionais e das táticas empresariais, dentro de uma empresa específica, e através dos negócios dentro de uma cadeia de suprimentos, com o 
objetivo de melhorar o desempenho em longo prazo das empresas individuais e da cadeia de suprimentos como um todo. (Mentzer et al., 2001, p. 18).

No contexto brasileiro, o tema Sustentabilidade em Cadeias de Suprimento está em pleno crescimento, pois, segundo Silva et al. (2015), as publicações têm aumentado (especialmente em conferência, e lentamente em periódicos). Assim, é válido desenvolver pesquisas que visam ampliar a discussão teórica no país. Nesse sentido, pesquisas como as de Abdala e Barbieri (2014), Carvalho e Barbieri (2013), Neutzling e Nascimento (2014), Silva e Nascimento (2016) têm demonstrado esforço em evidenciar aspectos locais, em consonância com o que apresentaram Gonçalves-Dias et al. (2012) sobre a necessidade de se buscar ressaltar o que é genuinamente brasileiro. Além disso, pode-se buscar aproximar discussões internacionais ao contexto nacional (como Brito \& Berardi, 2010), sempre na visão de avançar nas discussões, por vezes pouco consideradas.

Dentre os resultados da integração de práticas socioambientais nas organizações, está a conquista da vantagem competitiva/ colaborativa, debatida na área. Alguns exemplos de diferenciação das empresas podem ocorrer com o desenvolvimento de "melhores práticas" de negócios ou novos modelos de negócios (Epstein \&Roy, 2001), redução de custos (Bansal, 2005), legitimação social (Srivastava, 1995), entre outros. No entanto, a principal razão para a criação das vantagens competitivas advém da integração e dos relacionamentos interorganizacionais colaborativos que emergem nas cadeias (Brito \& Berardi, 2010; Chen \& Paulraj, 2004; Gold et al., 2010; Mohr \& Sengupta, 2002; Wolf, 2011).

Nesse sentido, as vantagens competitivas são discutidas como um resultado a partir do desenvolvimento de recursos e capacidades específicos quando as empresas inserem uma gestão socioambiental nos negócios. No mesmo sentido, emerge a vantagem colaborativa como vertente passível de aplicação no contexto de SCS. Considerando essa perspectiva, para a identificação de bases teóricas para sustentabilidade 
em cadeias de suprimento, inicialmente, é necessário entender as visões (VBR e VR) para então propor uma lógica mais prática de atuação. Vale salientar que são muitas as possibilidades de discussão teórica e, nesse caso, a seleção foi condicionada a uma primeira aproximação. Busca-se, a partir dessa visão de SCS e recursos e capacidades, propor uma linha de estudo que facilite novas pesquisas e discussões em uma perspectiva de aprofundamento teórico.

\subsection{Gestão estratégica a partir de recursos e capacidades: conceitos e discussões}

No ambiente organizacional, um dos principais desafios é desenvolver modelos de gestão capazes de conquistar e manter vantagens competitivas no mercado. Todavia, começam a emergir discussões que consideram o papel do comportamento colaborativo interorganizacional como propulsor de competitividade. Existem correntes que discutem a forma como as empresas se portam em relação ao seu ambiente externo (Porter, 1980) e outras (como a Teoria dos Recursos) que discutem essas vantagens, dando ênfase para os recursos e capacidades internos possuídos pelas empresas como determinantes das oportunidades existentes no ambiente externo e que seriam os grandes diferenciais e também propulsores de vantagens competitivas (Penrose, 1959).

Não cabe a indicação normativa sobre qual dessas perspectivas apresenta-se como a mais adequada, no entanto, a Visão Baseada em Recursos aborda as firmas numa visão de que estas se constituem em um amplo "feixe de recursos" (Penrose, 1959) que estabelecem condições únicas e primordiais para a diferenciação e, consequentemente, a sobrevivência da empresa no mercado. Segundo Vasconcelos e Cyrino (2000), os postulados de Penrose (1959) representaram uma mudança significativa nas discussões sobre os processos de expansão das firmas. Portanto, a VBR parte da premissa de que a combinação dos recursos das firmas influencia na determinação de estratégias que buscam a diferenciação. 
Os recursos e capacidades criados ao longo do tempo de forma particular influenciam o desempenho das firmas, uma vez que não podem ser adquiridos completamente pelos concorrentes. Essa condição poderia gerar um desempenho econômico diferenciado, logo, vantagem competitiva (Amit \& Schoemaker, 1993; Barney, 1991; Peteraf, 1993; Wernerfelt, 1984). Para Barney (1991, p. 102), a vantagem competitiva é conquistada "quando a firma implementa uma estratégia capaz de criar valor, a qual não está sendo simultaneamente implementada por qualquer outro concorrente atual ou potencial e, quando outras firmas não são capazes de duplicar os benefícios desta estratégia".

Asustentação da vantagem competitiva, nesse caso, está associada à impossibilidade de imitação dos recursos e, consequentemente das estratégias para alcançar a vantagem (Barney, 1991; Teece et al., 1997). Ou seja, está relacionada ao desenvolvimento de uma posição estratégica que diferencia sua forma de atuação dos demais participantes de um mesmo setor econômico.

Para uma compreensão mais efetiva dessa forma de diferenciação, é necessária uma visão conceitual tanto de recursos quanto de capacidades. Tratando-se da definição do que são recursos para a VBR, segundo Wernerfelt (1984, p. 172), "recursos são qualquer coisa que pode ser pensada na forma de força ou fraqueza de uma firma". Mais precisamente, recursos poderiam ser definidos como aqueles ativos (tangíveis e intangíveis) que estão ligados semipermanentemente à firma, como por exemplo: marcas, conhecimento tecnológico, emprego de capacidades e conhecimentos pessoais, contatos comerciais, maquinário, procedimentos eficientes, capital, dentre outros.

Na visão de Barney (1991, p. 101), os recursos das firmas "incluem todos os ativos, capacidades, processos organizacionais, atributos, informação e conhecimentos controlados pela firma". Esses recursos são classificados em três grupos: i) recursos de capital físico: tecnologias, equipamentos, localização geográfica e acesso a matérias-primas; ii) recursos de capital humano: experiência, posicionamento crítico, inteligência, relacionamento e insights de gestores e colaboradores da 
firma; e iii) recursos de capital organizacional: estrutura de comunicação formal, planejamento formal e informal, controle, coordenação de sistemas, como também as relações informais entre grupos dentro da firma e com seu ambiente externo.

Segundo Barney (1991), nem todos os recursos terão necessariamente relevância estratégica para as firmas, mas é sob esse conjunto, e de acordo com o seu gerenciamento, que alguns se destacarão e se combinarão de forma a gerar valor para a firma.

A partir das definições clássicas do que são recursos e capacidades, entende-se que são condições básicas para o alcance de vantagens, como descrito por Barney (1991): valiosidade, "rarabilidade", heterogeneidade, imperfeita imitabilidade (a partir das condições históricas de formação destes recursos, path dependence, ou trajetória, ambiguidade causal e complexidade social) e por possuírem uma estrutura de organização diferenciada (Barney, 1991; Barney \& Hesterly, 2007). Portanto, as premissas da VBR se pautam muito fortemente da busca que as empresas deveriam ter na diferenciação de seus recursos e capacidades com relação às características únicas. Nesse aspecto, Dierickx e Cool (1989) salientam a importância de conhecimento e aprendizado internos à empresa e acumulados ao longo do tempo, tornando-os inimitáveis.

Já na perspectiva de Grant (1991), os recursos são divididos em financeiros, físicos, humanos, tecnológicos, organizacionais e reputacionais. Além disso, as capacidades existentes nas firmas referemse à "habilidade de um conjunto de recursos de realizar uma tarefa ou atividade" (Grant, 1991, p. 119). O que, segundo o autor, não caracteriza a simples união de alguns recursos, mas a complementaridade entre esses e a possibilidade de filtrar e decidir sobre a utilização ou não de recursos no desenvolvimento de uma determinada prática organizacional.

Nesse sentido, destacam-se dois aspectos cruciais nas proposições de Grant (1991). Primeiramente, dentre os conjuntos de recursos, a relevância é dada aos intangíveis considerando as habilidades pessoais que, segundo o autor, "são provavelmente, os mais importantes recursos 
estratégicos que a firma possui" (Grant, 1991, p.119). Segundo o autor, recursos e capacidades intangíveis têm o potencial de serem mais duráveis do que os recursos sob os quais elas se formaram, devendo receber maior ênfase da gestão.

Já o segundo aspecto está na importância que o autor dá ao papel da cooperação e coordenação dos conjuntos de recursos e das capacidades da firma. Segundo Grant (1991), dependendo do grau de complexidade de coordenação do conjunto de recursos e capacidades possuído pelas empresas, dificilmente tais modelos serão imitados, dada a falta de pleno conhecimento e transparência dos concorrentes. A dificuldade de imitação estaria ligada também à dificuldade de mobilidade dos recursos possuídos relacionados à localização geográfica, informação imperfeita (heterogeneidade dos recursos e desconhecimento do potencial de produtividade, no caso principalmente de recursos humanos e recursos específicos da firma).

No contexto socioambiental, Silva e Balbino (2013) e Sousa Filho e Barbieri (2015) indicam que a responsabilidade socioambiental empresarial pode ser considerada como uma habilidade estratégica, uma vez que a partir dos recursos organizacionais é possível estruturar uma estratégia que aproxime a sustentabilidade da organização. Para o presente estudo, essa visão também permeia os argumentos.

No entanto, ao discutir as características de recursos e capacidades que tornariam as firmas competitivas, uma crítica é feita à VBR em relação à sua estrita orientação de olhar apenas internamente às empresas, tornando a análise dos recursos e da vantagem competitiva, de certa forma, estática (Doz, 1996; Felin \& Foss, 2005; Priem \& Butler, 2001). Com relação a isso, torna-se claro que é necessário observar uma perspectiva mais ampla que englobe as ações desenvolvidas internamente e transborde para os relacionamentos existentes com outras organizações. Assim, as firmas poderiam se tornar competitivas a partir dos relacionamentos de cooperação e colaboração estabelecidos com outras firmas atuantes no seu ambiente (Dyer \& Singh, 1998; Lavie, 2006). Essas proposições são trazidas pelos autores da Visão Relacional. 
A Visão Relacional trata da geração de recursos e capacidades oriundos de ligações idiossincráticas entre empresas pertencentes a uma cadeia de valor (Dushek, 2004; Dyer \& Singh, 1998). Segundo o conceito, ao compartilhar informações e capacidades, e a partir da frequência das transações de ativos específicos, as firmas passariam por um estágio de reciprocidade e transparência, em que novos conhecimentos seriam gerados (Horvath, 2001; Zacharia et al., 2011). Segundo Duschek (2004), a VR fundamenta-se na Visão Baseada em Recursos (VBR), mas considera também alguns elementos da Teoria dos Custos de Transação (TCT). Contudo, ainda que agregue alguns valores dessas abordagens, também as critica, visando superá-las no que diz respeito às formas de gerenciamento de recursos e obtenção das vantagens competitivas. Ao contrário dos postulados da VBR e TCT, para Dyer e Singh (1998), as principais fontes de competitividade estariam nas relações desenvolvidas fora dos limites das firmas e nas configurações de redes.

A Visão Relacional, portanto, é um conceito baseado na perspectiva de integração de estratégias entre empresas que possibilite o desenvolvimento de ligações idiossincráticas. A partir dos relacionamentos integrados é que as organizações se permitiriam a colaborar, trocar experiências e conhecimentos, possibilitando a criação de recursos e capacidades conjuntas e únicas (Dyer\&Singh, 1998; Lavie, 2006). O conceito da VR encontrou uma relação positiva entre o compartilhamento de ativos específicos (físicos, humanos e locacionais) e o desempenho dos parceiros em cadeias de suprimento. Para Dyer (1997), o investimento em ativos específicos estaria diretamente ligado aos custos de transação, uma vez que maior a especificidade dos ativos, maiores seriam os custos desta transação. No entanto, uma estrutura de coordenação diferenciada poderia influenciar nos custos da parceria ao basear-se em comprometimento, compartilhamento de informações e investimentos para o desenvolvimento de novos ativos.

Dessa forma, àmedida que os relacionamentos interorganizacionais atingissem o desenvolvimento de conhecimento conjunto e geração de 
novos recursos e capacidades, maiores seriam os níveis de integração das relações. O que a VR visa propor é que, fundamentalmente:

[...] as rendas relacionais são possíveis quando há alianças que combinam, trocam ou investem em ativos idiossincráticos, conhecimentos e recursos e/ou capacidades que empregam efetivamente mecanismos de governança, reduzindo assim, os custos de transação, ou, que permitem a realização de rendas através da combinação sinérgica de recursos, conhecimentos e capacidades entre as firmas. (Dyer \& Singh, 1998, p. $662)$.

Nesse sentido, Lavie (2006) salienta que tais rendas relacionais somente podem ser conquistadas a partir de recursos que são intencionalmente comprometidos e possuídos em conjunto pelos parceiros da aliança.

Portanto, a Visão Relacional destaca duas questões importantes para fazer com os relacionamentos atinjam o sucesso esperado. A primeira questão está relacionada à colaboração, especialmente quando há rotinas de trocas de conhecimento. Para isso, as empresas deveriam desenvolver condições de capacidades absortivas que thes permita identificar o valor de recursos externos, assimilá-los e combinálos aos seus recursos internos (Cohen \& Levinthal, 1990; Zacharia et al., 2011). Outros fatores essenciais na colaboração seriam desenvolver relações baseadas em transparência e reciprocidade, permitindo que as firmas compartilhem informações e know-how.

A outra questão importante está relacionada à governança. De acordo com Dyer e Singh (1998), as empresas precisam de garantias para apoiar suas transações. Por meio de uma governança eficaz, o comportamento oportunista pode ser evitado a partir de acordos autodeclarados informais e garantias que podem reduzir custos de transação e aumentar a confiança ao longo das relações. NesSe aspecto, a confiança é considerada um elemento essencial para proporcionar um melhor desempenho para os parceiros (Handfield \& Bechtel, 2002; Johnston et al., 2004). 
Na VR, assim como na VBR, haveria também mecanismos que preservariam as rendas relacionais. EsSes mecanismos referem-se à ambiguidade causal e à impossibilidade de replicação, já que: (1) os recursos e capacidades foram construídos a partir do aprendizado e conhecimentos interfirmas; (2) seria difícil replicar os mesmos atributos das empresas parceiras concorrentes; e (3) seria praticamente impossível replicar um ambiente complexo, social e institucional, que possui regras (formais e informais) e onde o oportunismo é controlado pela cooperação (Dyer \& Singh, 1998).

Dessa forma, enquanto a VBR traz a discussão da competitividade das firmas como um fruto do conjunto de recursos e capacidades internos, a Visão Relacional pressupõe que as firmas gerariam competências e recursos diferenciados e competitivos, a partir da interação e do desenvolvimento de relacionamentos interorganizacionais.

De acordo com as discussões que foram até então apresentadas, inicialmente, torna-se possível identificar uma convergência na utilização das duas abordagens voltadas para os recursos e capacidades, bem como uma linha de discussão totalmente compatível com a visão de sustentabilidade em cadeias de suprimento. Quando há o desenvolvimento de estratégias socioambientais em uma organização - e, por consequência, junto à sua cadeia de suprimento -, é possível compreender a relação direta entre os recursos e capacidades com o objetivo estratégico final. A partir da lógica de convergência entre os tópicos integrantes dessa discussão teórica, torna-se facilitado aproximar os temas, e com isso desenvolver uma visão integrativa entre sustentabilidade e práticas internas e externas a uma organização.

\section{Recursos e capacidades para a Sustentabilidade em Cadeias de Suprimento: insights para uma base teórica e prática}

No contexto da sustentabilidade, a VBR e a VR podem ser utilizadas para advogar em favor das vantagens que a inserção de 
valores sustentáveis traria para as organizações. Primeiramente, Hart (1995) propôs a conjunção dos pressupostos da VBR com a dimensão ambiental através da abordagem da Natural-Resource-BasedView, ou, em português, Visão Natural Baseada em Recursos. A partir da abordagem, o autor discute que o desenvolvimento de estratégias voltadas para a prevenção da poluição, gestão do ciclo de vida dos produtos e, de uma forma geral, para o desenvolvimento sustentável geraria políticas de melhorias contínuas, integração com stakeholders, redesenho de sistemas de produção mais responsáveis ambientalmente e uma visão ampla de tendências com as questões de sustentabilidade.

Essas ações levariam as empresas a obter custos produtivos menores, melhoria de tecnologias, aquisição de matérias-primas com menor impacto ambiental, dentre outros resultados. Além de aspectos de ganhos financeiros de curto prazo, a mudança de visão em relação às questões ambientais, por meio do engajamento e da interconectividade entre as ações práticas, guia a empresa a obter um desempenho superior na administração de problemas e responsabilidades ambientais. Devido às suas trajetórias, as firmas estariam criando uma condição competitiva diferenciada frente aos concorrentes (Hart, 1995).

No mesmo sentido, Sharma e Vredenburg (1998) defendem que algumas capacidades desenvolvidas por uma organização ambientalmente proativa permitiriam uma habilidade para balancear objetivos econômicos com ambientais, podendo usufruir de vantagens competitivas para a firma e soluções positivas para o meio ambiente. No Brasil, algumas discussões têm sido desenvolvidas, dentre as quais se pode ressaltar a proposta da Estratégia Socioambiental Baseada em Recursos (ESBR). Apresentada sob esse formato por Sousa Filho e Barbieri (2015), a ESBR é uma vertente que busca aproximar as discussões de VBR e VR da responsabilidade socioambiental (RSA), a partir da visão de uma empresa. Já Silva e Balbino (2013) discutem sobre a formação de vantagem competitiva sustentável a partir da VBR e também relacionada à RSA. Percebe-se, com isso, que existe uma potencialidade de discussão nesse tema. 
Com o passar do tempo, autores passaram a relacionar a VBR com práticas ambientais ao longo de cadeias de suprimento, no que tange às possibilidades de criação de recursos e capacidades geradores de vantagens competitivas (Bowen et al., 2001; Carter \& Carter, 1998; Förstl et al., 2010; Gavronski et al., 2011; Vachon \& Klassen, 2006), assim como a Visão Relacional sobre vantagens colaborativas, diante da importância do desenvolvimento de relacionamentos interorganizacionais (Chen \& Paulraj, 2004; Gold et al., 2010, Paulraj, 2011). No entanto, vale ressaltar que, para a presente discussão, trazse a abordagem da sustentabilidade em suas diferentes dimensões, não especificamente relacionado ao ambiental. Com isso, torna-se clara a possibilidade de sustentabilidade em cadeias de suprimento com a utilização das abordagens selecionadas, principalmente pelos estudos antes desenvolvidos.

Segundo Paulraj (2011), a sustentabilidade na gestão de suprimento permite combinar sinergicamente recursos e capacidades e formar rotinas de trocas idiossincráticas. Isso também poderia facilitar a informação e compartilhamento de conhecimento entre os parceiros. Já Gold et al. (2010) argumentam que a VR melhor explicaria o processo de criação de vantagens competitivas (e por vezes colaborativas) a partir da geração de recursos e capacidades interorganizacionais os quais seriam particularmente difíceis de replicar pelos concorrentes. Argumenta-se nesta discussão que é necessária uma convergência entre as visões e alinhamento à SCS, uma vez que tanto o interno como o externo precisam estar integrados para a sustentabilidade e aproximados à estratégia.

Justifica-se essa perspectiva, pois segundo Golicic e Smith (2013), para uma organização, é necessário considerar tanto as suas ações como o impacto de sua cadeia de suprimento nas atividades organizacionais. Com o impacto existente de uma organização na sociedade, para que a sustentabilidade seja praticada de fato, as práticas internas e as de seus envolvidos devem ser consideradas na elaboração da estratégia. Portanto, no sentido de ressaltar uma perspectiva prática para a presente discussão, busca-se identificar elementos que podem ser 
considerados como integrantes do processo de elaboração estratégica. Com isso, tem-se na responsabilidade socioambiental uma capacidade organizacional que favorece que se torne latente uma contribuição para a sustentabilidade.

No entanto, ainda que as situações ganha-ganha ao longo da cadeia de suprimento sejam identificadas, as condições de quando e como elas podem ser conquistadas ainda estão na fase inicial do seu desenvolvimento (Seuring \& Müller, 2008), bem como quais seriam os elementos a serem investigados na relação de recursos e capacidades geradores de vantagens competitivas. Assim, a discussão trazida possui argumentos mais focados no relacionamento, mas com base fundamentada na VBR, ou seja, na discussão clássica de recursos e capacidades. Como é possível observar, toda essa relação envolve uma eficiência estratégica e a integração entre os atores ao longo da cadeia, sendo possível considerar a convergência entre o interno e o externo.

Como observado na Figura 1, a influência das visões relacional e baseada em recursos faz emergir um conjunto de recursos e capacidades para a sustentabilidade em cadeias de suprimento e, potencial, vantagem competitiva/colaborativa. A utilização desses recursos e capacidades emergentes traz a visão prática resultante desta discussão teórica no sentido de demonstrar às estratégias organizacionais, direcionadores iniciais sobre como lidar com o tema. Entende-se que recursos e capacidades, em sua maioria, são inerentes à atividade desenvolvida por uma organização específica; com isso, esta seria um indicativo do que a literatura pode trazer e contribuir para esse contexto mais prático. 
Figura 1 - Recursos e capacidades identificados para SCS

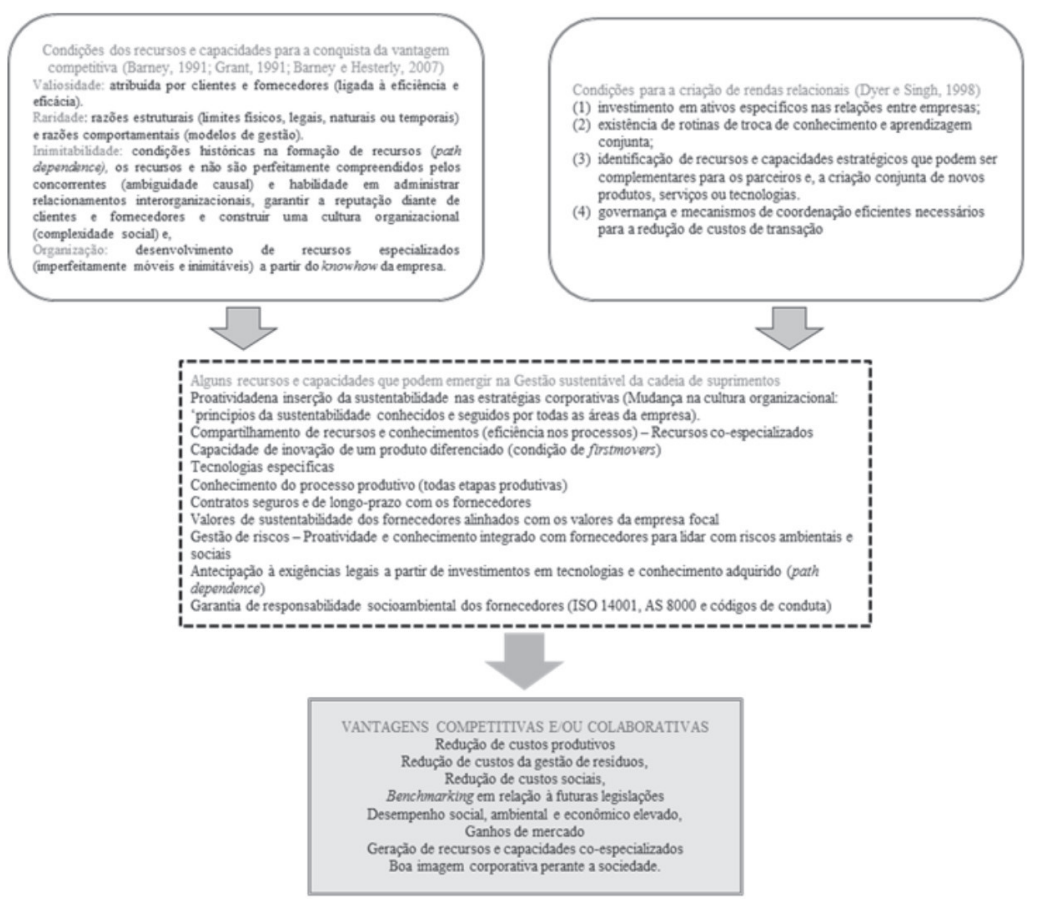

Fonte: Elaborada pelos autores.

A partir dessa discussão, podem-se ressaltar alguns dos pontos identificados, dentre os quais: pró-atividade, conhecimento do processo produtivo, contratos seguros e de longo prazo, tecnologias específicas e gestão de riscos. São recursos e capacidades que facilitam a sustentabilidade em cadeias de suprimento. Como identificado na Figura 1, a maioria pode ser classificada como intangível, o que está totalmente alinhado com a discussão de ESBR e, portanto, demonstra a existência de um direcionamento organizacional para lidar mais com o que é intangível. São raras as discussões que demonstrem a aplicabilidade desta afirmação, todavia a partir da literatura sobre o tema há uma 
tendência dos autores em indicar e salientar os recursos intangíveis como um dos mais importantes aspectos para a SCS.

Segundo Skjoett-Larsen (2003), a confiança entre uma empresa e seus fornecedores poderia ser usada como exemplo de um recurso intangível interorganizacional. Kogg e Mont (2012) argumentam também sobre as vantagens que existem nos relacionamentos colaborativos (mesmo que seja necessário certo tempo). Elas são explicadas justamente pelos elementos da visão relacional: governança mais eficaz e vantagem competitiva interorganizacional, tais como ativos específicos de relação, compartilhamento de capacidades e recursos complementares. Dessa forma, o aspecto relacional é tido como vital para que estratégias socioambientais sejam aplicadas em cadeias de suprimento e haja uma integração dos parceiros (Gold et al., 2010; Markley \& Davis, 2007; Pauraj, 2011).

Para a inserção de sustentabilidade a partir da visualização dos relacionamentos interorganizacionais, podem ser geradas competênciaschave e recursos internos voltados para a construção de capacidades na gestão de suprimento, na gestão estratégica de compras, bem como na pró-atividade socioambiental que facilita a conquista das vantagens competitivas, à medida que tal configuração tornar-se-ia única e inimitável (Gold et al., 2010; Markley \& Davis, 2007). Além disso, outros elementos destacam-se também, como o trabalho integrado em equipes, compreensão das questões ambientais entre as equipes de compras, bem como a capacitação técnica e as políticas globais de compra e procedimentos como alavancadores de uma série de vantagens (Gold et al., 2010).

O estabelecimento das relações de confiabilidade e compartilhamento de estratégias de sustentabilidade ao longo destas cadeias possibilita a criação de vantagens competitivas advindas não somente dos aspectos operacionais de eficiência e custos, mas também a partir da colaboração, permitindo a criação de novos conhecimentos e competências, melhoria das habilidades, convergindo em recursos e capacidades coespecializados (Gold et al., 2010; Gimenez et al., 2012; 
Markley \& Davis, 2007; Rao \& Holt, 2005). Entende-se a governança como necessária na coordenação dos relacionamentos. Na gestão da cadeia de suprimento, a governança é salientada como uma questão altamente relevante para promover confiança, reduzir riscos e proporcionar inovação (Zhu et al., 2007).

A maneira com que a governança para sustentabilidade em cadeia de suprimento é estabelecida sobre os relacionamentos influencia também na geração dos recursos e competências criadores de vantagens competitivas. A governança das estratégias socioambientais aplicadas aos relacionamentos da cadeia (com ênfase, principalmente, nos fornecedores) pode ser fundamental na troca e cocriação de recursos e capacidades valiosos entre as empresas. A integração e a socialização ressaltadas por Cousins e Menguc (2006), podem ser consideradas elementos importantes neste contexto, além da consideração das diferentes configurações em que a governança pode se estabelecer, influenciando no objetivo final de geração de vantagens na cadeia.

Além da influência da governança na possibilidade de geração de ganhos, estariam também as empresas subjugadas à adoção de uma postura diferenciada para que estes ganhos se efetivem, com destaque à responsabilidade e à colaboração. A responsabilidade para a sustentabilidade envolve o comprometimento interno das organizações, ao tratar da capacidade para inovar e orientação gerencial que influenciarão as estratégias individuais e posteriormente as que serão desenvolvidas em nível de cadeia. Já a colaboração para a sustentabilidade pode ser determinada como a externalização do comprometimento das organizações em prol das estratégias socioambiental na cadeia. Nesse aspecto, é essencial a adequação conjunta dos atributos tecnológicos para que os processos operacionais e de troca das informações e conhecimentos ocorra.

Diante da discussão realizada, objetiva-se mostrar a possibilidade de conversão e a compreensão de como recursos e capacidades podem facilitar a sustentabilidade em cadeias de suprimento, ou seja, na maneira como esses são integrados ao contexto estratégico. Portanto, 
ao relacionar a perspectiva da VBR e da VR à SCS pode se ter uma oportunidade de explicar melhor como elementos organizacionais internos à empresa, mediante mecanismos de governança apropriados, podem ser integrados em relacionamentos colaborativos nas cadeias de suprimento. Isso pode permitir que empresas e seus fornecedores utilizem-se de recursos e capacidades próprios, mas desenvolvam conhecimentos e habilidades conjuntas a partir de ativos relacionais específicos e rotinas de compartilhamento de recursos e capacidades complementares.

\section{Considerações finais}

A proposta deste estudo esteve em sugerir um caminho inicial de identificação de elementos considerados recursos e capacidades importantes no desenvolvimento de uma gestão socioambiental, e consequentemente, na geração de vantagens competitivas para as empresas. Essa perspectiva está em consonância com o apresentado por Silva e Balbino (2013,), os quais consideram que ao alinhar o novo objetivo das organizações, qual seja: direcionar suas atividades em vistas ao desenvolvimento sustentável à gestão estratégica organizacional, os firstmovers conseguirão sustentar suas vantagens competitivas desenvolvendo aspectos-chave ao seu posicionamento no mercado.

Vale salientar que o foco dessa perspectiva pode não estar apenas e necessariamente na lógica de competição, apesar da utilização de conceitos que favorecem essa lógica, mas pode emergir mais claramente a visão de cooperação, ou ainda, coopetição.

A partir da discussão, identifica-se a contribuição direta de duas abordagens teóricas (VBR e VR), o que pode ser considerado como um ponto de partida para a construção de bases teóricas para a compreensão da sustentabilidade em cadeias de suprimento. Todavia, vale ressaltar que outras abordagens - como a de capacidades dinâmicas - também podem contribuir diretamente com essa proposta. Como indicado por Pagell e Schevchenko (2014), caso certas bases consistentes não sejam desenvolvidas, a sustentabilidade em cadeias de suprimento não 
tem futuro tanto para a pesquisa como na prática, o que vai diretamente contrário ao que se tem de estudos. Com isso, identifica-se este ensaio teórico em si como uma contribuição positiva, um novo passo para avançar no tema.

Em uma perspectiva de base prática, à medida que se apresentam recursos e capacidades que podem contribuir para a sustentabilidade em cadeias de suprimento, como realizado no tópico anterior, isso favorece a incorporação de ações dentro de empresas internamente e em relação com sua cadeia de suprimento. As empresas podem se basear nesses fatores destacados tanto para elaborar novas estratégias socioambientais com o foco em cadeias de suprimento como ter referência sobre qual seria os potenciais recursos e capacidades possíveis para então aprofundamento de quais caminhos poderiam nortear suas ações organizacionais, principalmente aquelas voltadas para a sustentabilidade. Com isso, recomenda-se que para pesquisas futuras sejam utilizados recursos e capacidades como base de identificação de uma possível inserção de sustentabilidade em cadeias de suprimento, o que pode ratificar os argumentos aqui utilizados. Além disso, sugerese o aprofundamento de discussão sobre a visão estratégica, seja no sentido tradicional, considerando as relações de mercado, ou mesmo a utilização de uma abordagem flexível, como a estratégia como prática.

\section{Referências}

Abdalla, E. C. \& Barbieri, J. C. (2014). Determinants of Sustainable Supply Chain: an analysis of mensuration models of pressures and socio-environmental practices. Journal of Operations and Supply

Chain Management, São Paulo, Vol. 7, No 2, pp.110-122.

Ahi, P. \& Searcy, C. (2013). A comparative literature analysis of definitions for green and sustainable supply chain management, Journal of Cleaner Production, Vol. 52, pp. 329-341.

Amit, R. \& Schoemaker, P. J. H. (1993). Strategic assets and organizational rents. Strategic Management Journal, Chicago, Vol. 14, No 1, pp.33-46, 
Ashby, A.; Leat, M. \& Hudson-Smith, M. (2012). Making connections: a review of supply chain management and sustainability literature, Supply Chain Management: An International Journal, Vol. 17, No 5, pp. 497-516.

Bansal, P. (2005). Evolving sustainably: A longitudinal study of corporate sustainable development. Strategic Management Journal, Chicago, Vol. 26, No 3, pp. 197-218.

Barney, J. B. (1991) Firm resources and sustained Competitive Advantage. Journal of Management, New York Vol. 17, No. 1, pp. 99-120.

Barney, J. B. \& Hesterly, W. S. Administração Estratégica e Vantagem Competitiva. São Paulo: Prentice Hall, 2007.

Beske, P. (2012). "Dynamic capabilities and sustainable supply chain management", International Journal of Physical Distribution \& Logistics Management, Vol. 42, No 4, pp. 372-387.

Beske, P. \& Seuring, S. (2014). Putting sustainability into supply chain management, Supply Chain Management: an international journal, Vol. 19, No 3, pp. 322-331.

Bowen, F. E.; Cousins, P. D.; Lamming, R. C. \& Faruk, A. C. (2002). Horses for courses: explaining the gap between the theory and practice of green supply. Greener Management International, Vol. 35, pp.41-60.

Brito, R. P. \& Berardi, P. C. (2010). Vantagem Competitiva na Gestão Sustentável da Cadeia de Suprimentos: um metaestudo. Revista de Administração Eletrônica - RAE, Vol. 50, No 2, pp.155-169.

Carter, C. R. \& Rogers, D. S. (2008). A framework of sustainable supply chain management: moving toward new theory. International Journal of Physical Distribution \& Logistics Management, Vol. 38, No. 5, pp. 360-387. 
Carvalho, A. P. \& Barbieri, J. C. (2013). Inovações Socioambientais em cadeias de suprimentos: um estudo de caso sobre o papel da empresa focal, RAl - Revista de Administração e Inovação, São Paulo, Vol. 10, No 1, pp. 232-256.

Chen, I.J. \& Paulraj, A. (2004); Towards a theory of supply chain management: the constructs and measurements. Journal of Operations Management. Vol. 22, No. 2, pp.119-150

Cohen, W. \& Levinthal, D. (1990). Absorptive capacity: a new perspective on earning and innovation. Administrative Science Quarterly, Vol. 35, pp. 128-152.

Cousins, P. \& Menguc, B. (2006). "The implications of socialization and integration in supply chain management". Journal of Operations Management, Vol. 24, No. 5, pp. 604-620.

Dierickx, I. \& Cool, K. (1989). Asset stock accumulation and sustainability of competitive advantage. Management Science. Catonsville Vol. 35, No 12, pp. 1504-1511.

Dyer, J. H. \& Singh, H. (1998) The relational view: Cooperative strategy and sources of interorganizational competitive advantage. Academy of Management Review, Vol. 23, No. 4, pp.660-679.

Duschek S. (2004). Inter-firm resources and sustained competitive advantage. Management Revue. Vol. 15, No. 1, pp. 53-73.

Elkington, J. (1999). Triple bottom-line reporting: looking for balance. Australian CPA.

Epstein, M. J. \& Roy, M. (2001). Sustainability in action: identifying and measuring the key Performance drivers. Long Range Planning Journal, Oxford, Vol. 34, No 5, pp. 585-604.

Foerstl, K.; Reuter, C.; Hartmann, E. \& Blome, C. (2010). Managing supplier sustainability risks in a dynamically changing environment - 
Sustainable supplier management in the chemical industry. Journal of Purchasing \& Supply Management, Vol. 16, pp. 118-130.

Gavronski, I.; Klassen, R. D.; Vachon, S. \& Nascimento, L. F. (2011). A resource-based view of green supply management. Transportation Research Part E, Logistics and Transportation Review, Vol. 47, No. 6, pp. 872-885.

Gimenez, C.; Sierra, V. \& Rodon, J. (2012). Sustainable Operations: their impact on the triple bottom line. International Journal of Production Economics, Vol. 140, pp. 149-159.

Gold, S.; Seuring, S. \& Beske, P. (2010). Sustainable Supply Chain Management and Inter-Organizational Resources: a Literature Review. Corporate Social Responsibility and Environmental Management, Vol. 17, No. 4, pp. 230-245.

Golicic, S. L. \& Smith, C. D. (2013). A meta-analysis of environmental sustainable supply chain management practices and firm performance, Journal of Supply Chain Management, Vol. 49, No. 2, pp. 78-95.

Gonçalves-Dias, S. L. F.; Labegalini, L. \& Csillag, J. M. (2012). Sustentabilidade e cadeia de suprimentos: uma perspectiva comparada de publicações nacionais e internacionais, Produção, São Paulo, maio/agosto, Vol. 22, No. 3, pp.517-533.

Grant, R. M. (1991). The Resource-Based Theory Of Competitive Advantage: Implications of Strategic Formulation. California Management Review. Berkeley, CA, Vol. 33, No. 3, pp.114-134.

Halldórsson, Á., Kotzab, H. \& Skjott-Larsen, T. (2009). Supply chain management on the crossroad to sustainability: a blessing or a curse? Logistics Research, Vol. 1, No. 2, pp.83-94.

Hamel, G. \& Prahalad, C. K. (1995). Competindo pelo Futuro: estratégias inovadoras para obter o controle do seu setor e criar os mercados de amanhã. Rio de Janeiro: Campus. 
Handfield, R. B. \& Nichols, E. L. (1999). Introduction to Supply Chain Management. Upper Saddle River: Prentice-Hall.

Hart, S. (1995). A natural resource-based view of the firm. Academy of Management Review, Vol. 20, No. 4, pp.986-1014.

Horvath, L. (2001). Insight from the industry collaboration: the key to value creation in supply chain management. Supply Chain Management: An International Journal. Vol. 06, No. 05, pp.205-207.

Kogg, B. \& Mont, O. (2012). Environmental and social responsibility in supply chains: the practise of choice and inter-organisational management. Ecological Economics, Vol. 83, pp. 154-163.

Lavie, D. (2006). The competitive advantage of interconnected firms: an extension of the Resource-Based View. Academy of Management Review, Vol. 31, No. 3, pp. 638-658.

Meneghetti, F. K. (2011). O que é um ensaio teórico? Revista de Administração Contemporânea - RAC, Rio de Janeiro, Vol. 15, No. 2, pp. 320-332.

Mentzer, J. T., Dewitt, W., Keebler, J. S., Min, S., Nix, N. W., Smith, C. D. \& Zacharia, Z. G. (2001). Defining Supply Chain Management, Journal of Business Logistics, Vol. 22, No. 2, pp.1-25.

Mohr, J. J. \& Sengupta, S. (2002). Managing the paradox of inter-firm learning: the role of governance mechanisms. Journal of Business \& Industrial Marketing, Vol. 17, No. 4, pp. 282-301.

Neutzling, D. M. \& Nascimento, L. F. M. (2014). Integração na Gestão da Cadeias de Suprimento Sustentáveis: um abordagem teórica. Anais... XVII Simpósio de Administração da Produção, Logística e Operações Internacionais - SIMPOI, São Paulo: FGV, Brasil.

Pagell, M. \& Schevchenko, A. (2014). Why Research in Sustainable Supply Chain Management should have no Future, Journal of Supply Chain Management, Vol. 50, No. 1, pp. 1-32. 
Pagell, M. \& Wu, Z. (2009). Building a More Complete Theory of Sustainable Supply Chain Management Using Case Studies of 10 Exemplars. Journal of Supply Chain Management. Vol. 45, No. 2, pp. 37-56.

Paulraj A. (2011). Understanding the Relationships Between Internal Resources And Capabilities, Sustainable Supply Management and Organizational Sustainability. Journal of Supply Chain Management. Vol. 47, No 1, pp. 1-33.

Penrose, E. (1959) The theory of the growth of the firm. Oxford: Oxford University.

Peteraf, M. A. (1993) The Cornerstones of competitive advantage. Strategic Management Journal, Chicago, Vol. 14, No. 3, pp.179191.

Priem, R. L. \& Butler, J.E. (2001). Is the resource based "view" a useful perspective for strategic management research? Academy of Management Review, Vol. 26, No. 1, pp.57-66.

Rao, P. \& Holt, D. (2005). Do green supply chains lead to competitiveness and economic performance? International Journal of Operations \& Production Management, Vol. 25, No. 9, pp.898-916.

Seuring, S. \& Müller, M. (2008). From a literature review to a conceptual framework for sustainable supply chain management. Journal of Cleaner Production, Vol. 16, No. 15, pp. 1699-1710.

Sharma, S. \& Henriques, I. (2005). Stakeholder influences on sustainability practices in the Canadian forest products industry. Strategic Management Journal, Chicago, Vol. 26, pp. 159-180.

Shrivastava, P. (1995). Environmental technologies and competitive advantage. Strategic Management Journal, Chicago, Vol. 16, pp. $183-200$

Silva, M. E.; Neutzling, D. M.; Alves, A. P. F.; Dias, P.; Santos, C. A. F.; Nascimento, L. F. M. (2015). Sustainable Supply Chain Management: 
a Literature Review on Brazilian Publications, Journal of Operations and Supply Chain Management - JOSCM, São PauloVol. 8, No. 1, pp. 29-45.

Silva, M. E. \& Balbino, D. P. (2013). Criando Vantagem Competitiva Sustentável: a Responsabilidade Socioambiental Empresarial à luz da Visão Baseada em Recursos. Revista Ibero-americana de Estratégia, São Paulo, Vol. 12, No. 1, pp. 29-53.

Silva, M. E. \& Nascimento, L. F. M. (2016). (Re)Pensando a intersecção entre sustentabilidade e cadeia de suprimentos. Espacios (Caracas), Vol. 37, No 4.

Skjoett-Larsen, T.; Thernøe, C. \& Andresen, C. (2003) Supply chain collaboration theoretical perspectives and empirical evidence. International Journal of Physical Distribution \& Logistics Management, Vol. 33, No. 6, pp. 531-549.

Sousa Filho, J. M. \& Barbieri, J. C. (2015). Estratégia Socioambiental Baseada em Recursos e Ambiguidade Causal, Revista de Administração de Empresas - RAE, Vol. 55, No 6, pp. 699- 711.

Srivastava, S. K. (2007). Green Supply Chain Management: A Stateof-the-Art Review Literature Review. International Journal of Management Reviews, Malden, MA Vol. 9, No. 1, pp.53-80.

Vachon, S.; Klassen, R. D. (2006). Extending green practices across the supply chain: the impact of upstream and downstream integration. International Journal of Operations \& Production Management. Vol. 26, No. 7, pp. 795-821.

VasconceloS, F., Cyrino, A. (2000). Vantagem competitiva: os modelos teóricos atuais e a convergência entre estratégia e teoria organizacional. Revista de Administração de Empresas - RAE, São Paulo, Vol. 4, No. 40, pp.20-37.

Wernerfelt, B. (1984). A Resource-Based View of the Firm. Strategic Management Journal, Chicago, Vol. 5, pp.171-180. 
WCED - World Commission on Environment and Development. (1987). Report Our common future. Genebra. Disponível em: http://www. un-documents.net/wced-ocf.htm.

Wolf, J. (2011). Sustainable Supply Chain Management Integration: A Qualitative Analysis of the German Manufacturing Industry. Journal of Business Ethics, Vol. 102, pp. 221-235.

Zhu, Q.H.; Sarkis, J.; Lai, K.H. (2007). Green supply chain management: pressures, practices and performance within the Chinese automobile industry. Journal of Cleaner Production. Vol. 15, No. 11-12, pp. 1041-1052.

Artigo recebido em: 23/11/2015

Aprovado em: 05/02/2016 